\title{
Estudio comparativo de limitación por dolor en pacientes con osteoartrosis de rodilla operados de reemplazo total con prótesis Attune vs. prótesis PFC-Sigma
}

\section{Comparative study of pain limitation in patients with osteoarthrosis in total knee replacement with Attune prosthesis vs PFC-Sigma prosthesis}

\author{
Luis Manuel Alanís Blancas, ${ }^{*}$ Alberto Azcona Cervera, ${ }^{\star}$ Hugo Alejandro Rivera Ramírez*
}

\section{RESUMEN}

Introducción: El reemplazo articular de rodilla es el tratamiento de elección para la gonartrosis, una de las prótesis que con mayor frecuencia se colocan es la PFC-Sigma. Sin embargo, se ha reportado que $20 \%$ de los pacientes han referido limitaciones por dolor al realizar actividades cotidianas, por tal motivo se ha diseñado una nueva prótesis con el fin de mejorar la limitación y satisfacción de los pacientes con artroplastia de rodilla. Objetivo: Comparar la prótesis PFC-Sigma con la Attune, y demostrar que esta última tiene mejores resultados en cuanto a limitación por dolor y aceptación por parte de los pacientes. Material y métodos: Se trató de un estudio analítico retrospectivo que evaluó a 21 pacientes del Centro Médico ABC del 01 septiembre de 2013 al 15 de mayo de 2016 sometidos a artroplastia total de rodilla con prótesis PFC-Sigma (57\%) y prótesis Attune (43\%) con escala KOOS y EVERA. Resultados: Los pacientes operados con prótesis Attune tuvieron resultados satisfactorios en comparación con los pacientes operados con prótesis PFC-Sigma en la escala KOOS en el apartado de actividades cotidianas y actividades deportivas; en la escala EVERA no hubo diferencia significativa. Conclusiones: La prótesis Attune tuvo resultados satisfactorios en comparación con los pacientes operados con prótesis PFC-Sigma.

Palabras clave: Gonartosis, artroplastia, PFC-Sigma, Attune, prótesis.

Nivel de evidencia: III

\begin{abstract}
Introduction: Knee joint replacement is the treatment of choice for knee osteoarthritis, one of the prostheses that are most frequently placed is the PFC Sigma. However, it has been reported that $20 \%$ of patients have pain limitations when performing daily activities, for this reason a new prosthesis has been designed in order to improve the limitation and satisfaction of patients with knee arthroplasty. Objective: Compare the PFC-Sigma prosthesis with the Attune, and to demonstrate that Attune has better results in terms of pain limitation and acceptance by patients. Material and methods: This was a retrospective analytical study evaluating 21 patients of the ABC Hospital from September 1 of 2013 to May 15 of 2016, undergoing TKA with PFC-Sigma prosthesis (57\%) and Attune prosthesis (43\%). KOOS and NPIS scale. Results: The patients with Attune prosthesis had satisfactory results compared to the patients with PFC-Sigma prosthesis in the KOOS scale in the section of daily activities and sports activities, in the NPIS scale there was no significant difference. Conclusions: The Attune prosthesis had satisfactory results compared to patients operated on with PFC-Sigma prostheses.
\end{abstract}

Keywords: Gonartosis, total knee arthroplasty, PFC-Sigma, Attune, prosthesis.

Level of evidence: III

\section{* Ortopedia y Traumatología, Centro Médico ABC.}

Recibido para publicación: 09/03/2020. Aceptado: 01/06/2020.
Correspondencia: Dr. Alberto Azcona Cervera

Prol. Vasco de Quiroga Núm. 4001, Torre A, Piso 7, Consultorio 702, Col. Santa Fe, 05370. Alcaldía Cuajimalpa de Morelos, CDMX. Tel: 81647268 E-mail: azconaortopedia@gmail.com 


\section{INTRODUCCIÓN}

La osteoartritis es la más frecuente entre las patologías reumáticas con mayor evidencia en la historia. ${ }^{1,2}$ En los Estados Unidos afectó en 2005 a cerca de 26.9 millones de sus habitantes, de los cuales $13.9 \%$ eran adultos de 25 a 65 años y $33.6 \%$ eran mayores de 65 años. La osteoartritis de rodilla es la más frecuente, ${ }^{3,4}$ tiene una prevalencia de 37.4 (42.1 mujeres; 31.2 hombres) en mayores de 60 años, ${ }^{5}$ la incidencia es de 240 por 100,000 habitantes y aumenta de acuerdo con la edad hasta los 80 años. ${ }^{6,7}$ En 2009 se estimó un costo hospitalario para el tratamiento con reemplazo articular de rodilla de $\$ 28,500$ millones de dólares. ${ }^{8}$

La artrosis de rodilla primaria se encuentra entre los motivos frecuentes de consulta y de intervención quirúrgica en los centros hospitalarios de México. ${ }^{9,10}$ La relevancia y el impacto de su estudio residen en que se asocian con el incremento de la esperanza de vida de los pacientes que la han padecido y que han recuperado la movilidad articular posterior a procedimientos quirúrgicos. ${ }^{11,12}$

Los trastornos artrósicos además de estar relacionados con la edad afectan las articulaciones sinoviales, ${ }^{13,14}$ pueden generar cambios estructurales degenerativos en el cartílago, hueso subcondral, tejido sinovial, cápsula articular y tejidos blandos periarticulares. ${ }^{15,16}$ La osteoartritis se clasifica en primaria (idiopática) y secundaria (etiología conocida). ${ }^{17}$ Existen diversos factores que aumentan el riesgo de padecerla (Tabla 1);18-21 sin embargo, actualmente se desconoce su etiología. 22,23

El diagnóstico es un proceso que se realiza mediante criterios ya establecidos (Tabla 2) ${ }^{24,25}$

Para llevar a cabo una valoración radiológica se necesitan proyecciones en AP, lateral, axial de rótula y eje mecánico en búsqueda de osteofitos, disminu-

Tabla 1: Factores de riesgo de osteoartritis.

\begin{tabular}{|c|c|c|}
\hline Modificables & $\begin{array}{l}\text { Potencialmente } \\
\text { modificables }\end{array}$ & No modificables \\
\hline $\begin{array}{c}\text { Sobrecarga articular } \\
\text { Obesidad }\end{array}$ & $\begin{array}{l}\text { Trauma mayor } \\
\text { Defectos } \\
\text { propioceptivos }\end{array}$ & $\begin{array}{c}\text { Edad }>50 \text { años } \\
\text { Sexo femenino }\end{array}$ \\
\hline \multirow{4}{*}{$\begin{array}{l}\text { Debilidad muscular } \\
\text { Actividad física pesada } \\
\text { Nutrición (baja ingesta } \\
\text { de vitamina C) }\end{array}$} & Atrofia cuádriceps & Raza \\
\hline & Laxitud articular & Trastornos metabólicos \\
\hline & $\begin{array}{c}\text { Enfermedad } \\
\text { inflamatoria articular }\end{array}$ & Genética \\
\hline & & $\begin{array}{l}\text { Trastornos } \\
\text { congénitos }\end{array}$ \\
\hline
\end{tabular}

Tabla 2: Criterios para gonartrosis de American College of Rheumatology.

\begin{tabular}{c}
\hline Gonalgia (+ al menos tres) \\
Edad $>50$ años \\
Rigidez matutina $>30$ minutos \\
Crepitación ósea a los movimientos \\
Dolor a la presión sobre márgenes óseos de la articulación \\
Ausencia de evidencia de inflamación \\
Hipertrofia articular
\end{tabular}

ción del espacio articular, esclerosis, defectos óseos y calcificaciones de tejidos blandos y deformidades en varo o valgo. ${ }^{26-29}$

Los estudios realizados durante los últimos años se han enfocado en la insatisfacción de los pacientes sometidos a ATR, Bourne y colaboradores 12 reportaron un índice de insatisfacción entre 10 y $20 \%$ de los pacientes con ATR, siendo el dolor la principal limitación. Un estudio efectuado por la AARP (Association of American Retired Persons) en 2007 demostró que $58 \%$ de los pacientes con ATR presentaban dolor al arrodillarse y 38\% al hacer sentadillas. ${ }^{11}$ En 2012 Rothman y su equipo ${ }^{14}$ y otros estudios demostraron que $25 \%$ de los pacientes presentaban dolor anterior de rodilla a pesar de haber realizado adecuadamente fisioterapia. En los años 80 se creía que el arco de movilidad funcional para la rodilla debería ser de $90^{\circ} ;{ }^{30}$ sin embargo, las guías actuales refieren que debe ser de $120^{\circ}$ a $130^{\circ}$ para poder realizar actividades sin limitación. ${ }^{31,32}$

Se ha reportado que la prótesis PFC-Sigma tiene una vida media de 10 años en $98 \%$ de los casos, principalmente al efectuar movimientos rotatorios y al subir o bajar escaleras, esto se ha atribuido a la deficiencia del diseño de los implantes y al inadecuado balanceo que al con el tiempo llevan a la limitación de la flexión de la rodilla y a la sensación de inestabilidad. ${ }^{33-37}$

Todo lo mencionado anteriormente ha llevado a la creación de nuevos modelos para mejorar los componentes de la prótesis. ${ }^{38}$ En este sentido, en la presente investigación se plantea la necesidad de llevar a cabo un estudio de limitación por dolor y satisfacción sobre la prótesis Attune, en comparación con la prótesis previa PFC-Sigma para valorar los nuevos avances y sus resultados en cuanto al diseño de la nueva prótesis en los pacientes. Por tal motivo, se realizó el estudio evaluando por medio de la encuesta EVERA (escala verbal análoga del dolor) vía telefónica para evaluar el dolor residual posterior a la colocación de ambas prótesis. También se evaluó el cuestionario KOOS (Knee injury and Osteoarthritis 
Outcome Score) para conocer el grado de limitación que tienen los pacientes por dolor, sintomatología, actividades de la vida cotidiana, actividades deportivas y calidad de vida. La escala KOOS es de dominio público ${ }^{39}$ y se ha utilizado en diversos estudios para comparar limitaciones en patologías y procedimientos de la rodilla, entre ellas se encuentra la gonartrosis y la artroplastia. ${ }^{40-42}$ Por último, se preguntó a los pacientes si se volverían a someter al procedimiento en la rodilla contralateral como método para evaluar la aceptación de la prótesis.

La investigación realizada ha tenido la intención de conocer si esta nueva prótesis tiene menores limitaciones en cuanto a dolor y mejor satisfacción que las previas.

El objetivo del estudio es determinar que los pacientes con gonartrosis operados de reemplazo total con la prótesis Attune tienen menor limitación por dolor y satisfacción postquirúrgica en comparación con los pacientes operados con prótesis PFC-SIGMA.

\section{MATERIAL Y MÉTODOS}

La metodología empleada es de tipo cuantitativo; se basa en el uso de técnicas estadísticas, explicadas más adelante, para estudiar a una población específica a la que se denomina como «muestra» de pacientes del Centro Médico ABC sometidos a ATR con prótesis PFC-Sigma y prótesis Attune.

Se usa como técnica la entrevista por teléfono, para la cual se realiza un cuestionario donde se incluyen variables a analizar como:
- Variables independientes:

- Pacientes operados con prótesis PFC-Sigma.

- Pacientes operados con prótesis Attune.

- Variables dependientes:

- Escala visual análoga del dolor.

\section{Escala KOOS \\ (Knee injury and Osteoarthritis Outcome Score)}

Con cinco apartados: dolor, síntomas, actividades cotidianas, actividades deportivas, calidad de vida.

Pregunta: ¿Se operaría la rodilla contralateral si tuviese los mismos síntomas?

Para la selección de los integrantes de la muestra del presente estudio se han seguido los siguientes criterios de inclusión: pacientes operados en Centro Médico $\mathrm{ABC}$ Santa $\mathrm{Fe}$, pacientes sometidos a ATR primaria, haber colocado prótesis Sigma y Attune, sin retención de ligamento cruzado anterior, componente de tibia no rotatorio, diagnóstico de artritis reumatoide u osteoartrosis de rodilla, edad mayor de 45 años y haber sido operados por un médico ortopedista. Los criterios de exclusión son: pacientes sometidos a revisión de prótesis, diagnóstico de artrosis postraumática, pacientes sometidos a prótesis bilateral en un solo tiempo y cirugía efectuada en un tiempo menor de dos meses.

Se realizó una búsqueda retrospectiva dentro de la base de datos de pacientes operados, desde el 01 septiembre de 2013 hasta el 15 de mayo de 2016. To-

Tabla 3: Tabla demográfica.

\begin{tabular}{|c|c|c|c|c|c|c|c|c|c|}
\hline \multicolumn{5}{|c|}{ Grupo A } & \multicolumn{5}{|c|}{ Grupo B } \\
\hline Caso & Fecha Qx & Lado & Sexo & Edad & Caso & Fecha Qx & Lado & Sexo & Edad \\
\hline 1 & $23 / 09 / 2013$ & D & $\mathrm{F}$ & 80 & 1 & 08/07/2015 & I & $\mathrm{F}$ & 47 \\
\hline 2 & $11 / 11 / 2013$ & D & $\mathrm{F}$ & 55 & 2 & $10 / 08 / 2015$ & D & $\mathrm{F}$ & 65 \\
\hline 3 & $25 / 11 / 2013$ & D & $\mathrm{F}$ & 68 & 3 & $11 / 08 / 2015$ & D & $\mathrm{F}$ & 75 \\
\hline 4 & 06/01/2014 & D & $\mathrm{F}$ & 65 & 4 & 07/09/2015 & I & $\mathrm{F}$ & 66 \\
\hline 5 & 08/06/2014 & D & $\mathrm{F}$ & 71 & 5 & 04/10/2015 & I & $\mathrm{F}$ & 70 \\
\hline 6 & $24 / 10 / 2014$ & D & $\mathrm{F}$ & 84 & 6 & $19 / 10 / 2015$ & D & $\mathrm{F}$ & 75 \\
\hline 7 & 03/11/2014 & 1 & M & 61 & 7 & $29 / 02 / 2016$ & D & $\mathrm{F}$ & 78 \\
\hline 8 & $11 / 01 / 2015$ & I & $\mathrm{F}$ & 84 & 8 & 07/03/2016 & D & $\mathrm{F}$ & 72 \\
\hline 9 & $25 / 01 / 2015$ & I & $\mathrm{F}$ & 72 & 9 & $14 / 03 / 2016$ & D & $\mathrm{F}$ & 67 \\
\hline 10 & $08 / 03 / 2015$ & D & M & 83 & & & & & \\
\hline 11 & $26 / 04 / 2015$ & D & $\mathrm{F}$ & 70 & & & & & \\
\hline 12 & $12 / 06 / 2015$ & 1 & $\mathrm{~F}$ & 77 & & & & & \\
\hline \multicolumn{3}{|c|}{ Total } & & 72.5 & & & & & 68.3 \\
\hline
\end{tabular}


Tabla 4: Resultados PFC-Sigma.

\begin{tabular}{|c|c|c|c|c|c|c|c|c|}
\hline \multicolumn{9}{|c|}{ Grupo A } \\
\hline Caso & EVERA & Pregunta & $\begin{array}{l}\text { KOOS } \\
\text { dolor }\end{array}$ & $\begin{array}{l}\text { KOOS } \\
\text { síntomas }\end{array}$ & KOOS AC & KOOS AD & KOOS CV & $\begin{array}{c}\text { Total } \\
\text { KOOS }\end{array}$ \\
\hline 1 & 7 & No & 50 & 46 & 46 & 30 & 44 & 43.2 \\
\hline 2 & 1 & Sí & 75 & 82 & 81 & 65 & 75 & 75.6 \\
\hline 3 & 1 & Sí & 72 & 79 & 78 & 40 & 63 & 66.4 \\
\hline 4 & 1 & Sí & 86 & 79 & 85 & 55 & 75 & 76.0 \\
\hline 5 & 0 & Sí & 83 & 86 & 81 & 65 & 69 & 76.8 \\
\hline 6 & 2 & Sí & 78 & 86 & 85 & 65 & 69 & 76.6 \\
\hline 7 & 1 & Sí & 83 & 82 & 81 & 75 & 75 & 79.2 \\
\hline 8 & 2 & Sí & 83 & 89 & 81 & 70 & 69 & 78.4 \\
\hline 9 & 1 & Sí & 78 & 82 & 87 & 65 & 75 & 77.4 \\
\hline 10 & 4 & No & 81 & 89 & 78 & 65 & 69 & 76.4 \\
\hline 11 & 2 & Sí & 69 & 75 & 76 & 65 & 69 & 70.8 \\
\hline 12 & 2 & Sí & 83 & 86 & 78 & 75 & 75 & 79.4 \\
\hline Total & 2 & & 77 & 80 & 78 & 61 & 69.0 & 73.0 \\
\hline & & Sí 83\% & & & & & & \\
\hline
\end{tabular}

$A C=$ actividades cotidianas,$A D=$ actividades deportivas,$C V=$ calidad de vida

dos los pacientes fueron operados bajo isquemia, con abordaje de Insall, no se reportaron complicaciones durante el procedimiento quirúrgico. Se obtuvo un total de 31 casos de pacientes operados que fueron sometidos a los criterios de inclusión y exclusión: el grupo A con 16 pacientes con prótesis PFC-Sigma y el grupos B con 15 pacientes con prótesis Attune; sin embargo, debido a que algunos pacientes se rehusaron a responder el cuestionario, se eliminaron nueve pacientes, quedando un total de 12 para el grupo $\mathrm{A}$ y nueve para el grupo B, representados en la Tabla 3.

La entrevista telefónica se llevó a cabo del 07 de junio de 2016 al 11 de junio de 2016, la cual se inició preguntando la edad actual del paciente, seguida de la aplicación del cuestionario KOOS. Posteriormente, se les solicitó que calificaran ellos mismos el dolor que tuviesen con la escala visual análoga del dolor del 0 al 10. Por último, se les preguntó: si tuviese la misma sintomatología previa a la cirugía en la rodilla contralateral, ¿se volvería a operar? Cabe señalar que todos los datos fueron registrados en físico para su posterior recopilación y transcripción.

La escala KOOS es un cuestionario de dominio público ${ }^{26}$ que consta de cinco apartados que califican: dolor, síntomas, actividades cotidianas, actividades deportivas y calidad de vida. La respuesta de cada pregunta se calificó del 0 al cuatro según la respuesta, posteriormente se realizaron cálculos en cada apartado para escalar los resultados del 0 (con limi- taciones) al 100 (sin limitaciones) por cada apartado; además se calculó el promedio de los apartados para obtener una calificación global de KOOS.

$\mathrm{Al}$ reunir la información de los dos grupos se procedió a calcular la media de cada uno de los resultados de cada apartado para compararlos. El valor de «p» fue de 0.05 por el tamaño reducido de la muestra, en el caso de la escala EVERA y KOOS se utilizó la t de Student para obtener el valor de «p». Para la pregunta se empleó el test exacto de Fisher al tratarse de variables cualitativas en una muestra pequeña para obtener el valor de «p».

\section{RESULTADOS}

El grupo A tuvo 12 pacientes con dos hombres y 10 mujeres con una edad media de 72.5 años, el lado predominante fue el derecho, la fecha quirúrgica más antigua fue de dos años cinco meses y la más reciente fue de un año un mes (Tabla 3).

El grupo B tuvo nueve pacientes con $100 \%$ de mujeres, con una edad media de 68.3 años, el lado predominante fue el derecho, la fecha quirúrgica más antigua fue de 10 meses y la más reciente dos meses (Tabla 3).

Los resultados de cada grupo están expuestos en las Tablas 4 y 5 .

El dolor que refirieron los pacientes al realizar la entrevista telefónica fue registrado mediante la escala visual análoga del dolor, en el grupo A varió del grado 0 al 7 con una media de grado 2 (DE 1.86), en 
el grupo B del grado 0 al 2, con una media de 0.6 (DE 0.67 ) con un valor de «p» de 0.764 (IC-0.14 a 2.59).

La pregunta: si tuviese la misma sintomatología previa a la cirugía en la rodilla contralateral ise volvería a operar? En el grupo A 83\% respondió que sí y $17 \%$ respondió no, en el grupo B 89\% respondió que sí y $11 \%$ respondió que no, con una «p» de 1.0.

Para la escala KOOS en el apartado de dolor el grupo A tuvo una media de 77 ( $\mathrm{DE}=9.84)$, el grupo B mostró una media de $82(\mathrm{DE}=9.09)$ con un valor de «p» de 0.184 (IC 95\% = -12.95 a 2.67), el cual no fue estadísticamente significativo. El apartado de síntomas el grupo A tuvo una media de 80 $(\mathrm{DE}=11.54)$, el grupo B mostró una media de 81 $(\mathrm{DE}=8.96)$ con un valor de «p» de 0.79 (IC 95\% = -10.97 a 8.47), el cual no fue estadísticamente significativo. En las actividades cotidianas el grupo A tuvo una media de 78 ( $\mathrm{DE}=10.63)$, el grupo B mostró una media de $89(\mathrm{DE}=3.46)$ con un valor de «p» de 0.0082 (IC 95\% = -18.66 a -3.17), el cual sí fue estadísticamente significativo. En el apartado de actividades deportivas el grupo A tuvo una media de 61 $(\mathrm{DE}=13.5)$, el grupo B mostró una media de $74(\mathrm{DE}$
$=6.97)$ con un valor de «p» de 0.019 (IC 95\% = -23.0 a -2.28), el cual fue estadísticamente significativo. En cuanto a la calidad de vida el grupo A tuvo una media de 69 ( $\mathrm{DE}=8.74)$, el grupo B mostró una media de $72(\mathrm{DE}=8.23)$ con un valor de «p» de 0.491 (IC $95 \%$ $=-10.51$ a 5.24 ), el cual no fue estadísticamente significativo. En el promedio de todos los apartados el grupo A tuvo una media de $73(\mathrm{DE}=10.08)$, el grupo B mostró una media de $80(\mathrm{DE}=5.43)$ con un valor de «p» de 0.096 (IC 95\% = -14.3 a 1.27), el cual no fue estadísticamente significativo.

\section{DISCUSIÓN}

En el apartado de actividades cotidianas y actividades deportivas del cuestionario de KOOS las únicas estadísticamente significativas fueron con una «p» de 0.008 y 0.014 respectivamente. Cabe mencionar que los pacientes del grupo B reportaron menor tiempo de recuperación que el grupo A y que a pesar de esta situación tiene una tendencia a obtener medias más altas pese a no poseer una significativa estadística. La escala KOOS tuvo diferencias a fa-

Tabla 5: Resultados Attune.

\begin{tabular}{|c|c|c|c|c|c|c|c|c|}
\hline \multicolumn{9}{|c|}{ Grupo B } \\
\hline Caso & EVERA & Pregunta & $\begin{array}{l}\text { KOOS } \\
\text { dolor }\end{array}$ & $\begin{array}{l}\text { KOOS } \\
\text { síntomas }\end{array}$ & KOOS AC & KOOS AD & KOOS CV & $\begin{array}{c}\text { Total } \\
\text { KOOS }\end{array}$ \\
\hline 1 & 2 & Sí & 75 & 71 & 87 & 65 & 75 & 74.6 \\
\hline 2 & 1 & Sí & 86 & 93 & 91 & 75 & 75 & 84.0 \\
\hline 5 & 1 & No & 81 & 93 & 87 & 70 & 75 & 81.2 \\
\hline 6 & 0 & Sí & 89 & 86 & 91 & 85 & 69 & 84.0 \\
\hline 7 & 1 & Sí & 81 & 82 & 90 & 75 & 63 & 78.2 \\
\hline 8 & 0 & Sí & 78 & 71 & 84 & 70 & 69 & 74.4 \\
\hline 9 & 1 & Sí & 72 & 71 & 87 & 65 & 56 & 70.2 \\
\hline Total & 0.6 & $\begin{array}{l}\text { Sí } 89 \% \\
\text { No } 11 \%\end{array}$ & 82 & 81 & 89 & 74 & 72 & 80 \\
\hline
\end{tabular}

$A C=$ actividades cotidianas, $A D=$ actividades deportivas,$C V=$ calidad de vida.

Tabla 6: Medias por apartado en escala KOOS.

\begin{tabular}{ccccccc}
\hline Grupo & Dolor & Síntomas & $\begin{array}{c}\text { Actividades } \\
\text { cotidianas }\end{array}$ & $\begin{array}{c}\text { Actividades } \\
\text { deportivas }\end{array}$ & Calidad de vida & Total KOOS \\
\hline A & 77 & 80 & 78 & 61 & 69 & 73 \\
B & 82 & 81 & 89 & 74 & 72 & 3 \\
Diferencia & 5 & 1 & 11 & 12 & 3 \\
\hline
\end{tabular}




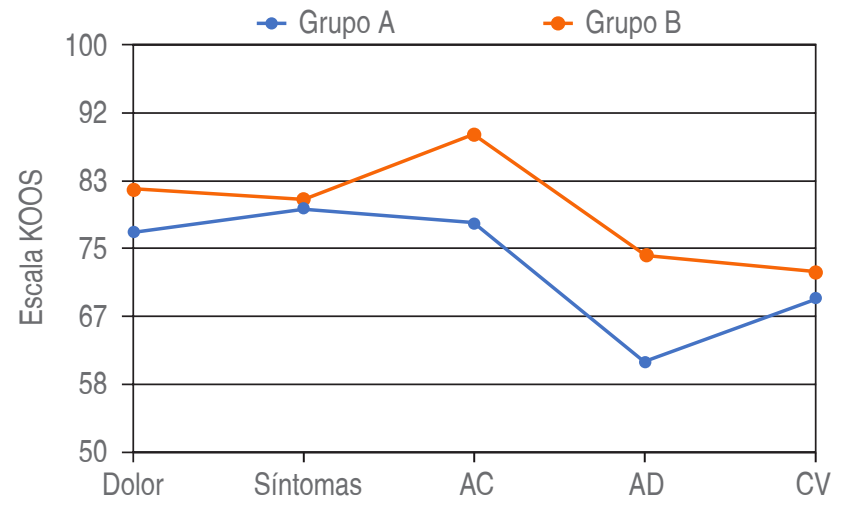

Figura 1: Medias escala KOOS.

$A C=$ actividades cotidianas,$A D=$ actividades deportivas,$C V=$ calidad de vida .

vor en la mayoría de sus apartados expuestos en la Tabla 6 y en la Figura 1.

En el apartado de dolor hubo una diferencia de cinco puntos a favor del grupo de la nueva prótesis, el grupo A tuvo una media de 77, el puntaje varió de 50 (caso 1) a 86 (caso 4), en el grupo B la media fue de 82 , el puntaje varió de 72 (caso 2) a 89 (caso 6). El resultado al comparar los dos grupos no fue estadísticamente significativo, «p» de 0.184. Sin embargo, el grupo de Attune tiene una tendencia a tener menos dolor que la PFC-Sigma. En el apartado de síntomas, el grupo A tuvo una media de 80, el puntaje varió de 46 (caso 1) a 89 (caso 10). El grupo B tuvo una media de 81, el puntaje varió de 71 (caso 1) a 93 (caso 2 y 5 ). En este grupo la diferencia de medias fue de uno a favor del grupo de Attune, por lo que no fue estadísticamente significativo con una «p» de 0.790. El apartado de actividades cotidianas fue estadísticamente significativo con una «p» de 0.008, la diferencia entre los grupos fue de 11 puntos a favor del grupo de Attune. En el grupo A, con una media de 78, el puntaje varió de 46 (caso 1) a 87 (caso 9). En el grupo B tuvo una media de 89 con un puntaje de 87 (caso 1 y 9) hasta 96 (caso 4), los reactivos que demostraron tener mayor limitación fueron el 16 (realizar trabajos pesados en casa) seguido del 1 y 2 (subir y bajar escaleras). En las actividades relacionadas al deporte, el grupo A tuvo una media de 62 con un puntaje que varió desde 40 (caso 1), hasta 75 (casos 7 y 12). En el grupo B la media fue de 74 con un puntaje de 65 (caso 1 y 9) hasta 85 (caso 6) la diferencia entre estos grupos fue de 12 puntos. Este apartado tuvo la diferencia más grande entre las medias, con una "p» de 0.014, siendo estadísticamente significativa a favor del grupo B. Los reactivos con mayor limitación fueron (por orden) el 3 (saltar), 2 (correr), y 4 (girar o pivotear). El apartado de calidad de vida el grupo A tuvo una media de 69 con un puntaje que varió de 44 (caso 1) a 75 (caso 2, 7, 9 y 12). En el grupo B la media fue de 72 con un puntaje que varió de 56 a 81, la diferencia entre los grupos fue de tres puntos, no fue estadísticamente significativa al tener una «p» de 0.491; sin embargo, con tendencia a favor del grupo B.

Al realizar el KOOS total, el grupo A tuvo una media de 73 , el puntaje varió de 43.2 (caso 1 ) a 79.4 (caso 12). El grupo B tuvo una media de 80 , varió de 70.2 (caso 9) a 85.8 (caso 4) al comparar los dos grupos la diferencia fue de siete puntos con tendencia a favor del grupo B, la «p» fue de 0.096, siendo no estadísticamente significativa. ${ }^{43,44}$

La EVERA no fue estadísticamente significativa. La diferencia varió de 0 a siete, algunos de los resultados de los casos no correspondían con el puntaje en el cuestionario KOOS, estos resultados se deben a que la escala de dolor es subjetiva y depende de las perspectivas y experiencias previas del paciente en cuanto al dolor, si comparamos el dolor entre los grupos en la escalas EVERA y KOOS, se puede observar que en el grupo A hay más dolor tanto en EVERA (2 vs. 0.6) como en KOOS (77 vs. 82) favoreciendo al grupo de Attune. Esto se puede respaldar con algunos estudios similares que han demostrado que los pacientes con prótesis Attune tienen mayor satisfacción que la PFC-Sigma en los primeros seis meses postquirúrgicos; sin embargo, al transcurrir dos años no hay diferencia significativa entre ellas.

La pregunta aplicada a todos los pacientes tiene la finalidad de saber la aceptación de la prótesis. En el grupo A 17\%, (dos pacientes) respondieron que no. El caso 10 comentó que no deseaba volverse a operar por su edad (83 años), ya que no se sentía apto para una nueva cirugía. En el grupo B 11\% correspondiente al caso 5 respondió negativamente por sentir que generó mucha molestia en su familia por la operación, aunque esta variable tampoco fue estadísticamente significativa, se puede notar que ambas prótesis tienen adecuada aceptación en los dos grupos.

En cuanto a las limitaciones de la presente investigación se puede comentar que el estudio cuenta con una cantidad reducida de pacientes debido a que la prótesis Attune ha salido al mercado y ha iniciado su colocación no hace mucho tiempo; esto también afecta al tiempo postquirúrgico del paciente para que pueda realizar fisioterapia que le permita una adecuada adaptación de la prótesis y hacer una mejor comparación. Otra limitación es que por la escasa cantidad de prótesis que se ha colocado, por los motivos ya mencionados, el médico aún se encuentra en la curva de aprendizaje de la colocación de la prótesis Attune. 


\section{CONCLUSIONES}

La prótesis Attune tuvo menor limitación que la prótesis PFC-Sigma en cuanto a la escala KOOS en el apartado de actividades cotidianas y actividades deportivas. El dolor global medido con las escalas EVERA y KOOS no mostró tener relevancia estadística ni la decisión de una reintervención en la rodilla contralateral; sin embargo, la tendencia de ambas es a favor de la prótesis Attune. Con respecto al objetivo se demostró que la prótesis Attune tiende a ser menos limitante y por lo tanto, es una mejor alternativa que su predecesora PFC-Sigma. Los pacientes operados con prótesis Attune tuvieron resultados satisfactorios en comparación con los pacientes operados con prótesis PFC-Sigma. Es necesario realizar estudios más amplios con mayor número de pacientes y médicos para evaluar de manera global la prótesis Attune, incluso se necesitaría revaluar a los pacientes en un periodo postquirúrgico más prolongado.

\section{BIBLIOGRAFÍA}

1. Góngora LH, Rosales CM, González I. Articulación de la rodilla y su mecánica articular. Medisan. 2003; 7 (2): 100-109.

2. Wieland H, Michaelis M, Kirschbaum B. Osteoarthritis-an untreatable disease? Nature Reviews Drug Discovery. 2005; 4 (4): 331-344.

3. Goodman S. Osteoarthritis. In: Yee A, Paget S, eds. Expert Guide to Rheumatology. Philadelphia, Pa.: American College of Physicians; 2005, pp. 269-283.

4. Lawrence R, Felson D, Helmick C. Estimates of the prevalence of arthritis and other rheumatic conditions in the United States: Part II. Arthritis Rheum. 2007; 58 (1): 26-35.

5. Heidari B. Knee osteoarthritis prevalence, risk factors, pathogenesis and features: part I. Caspian J Intern Med. 2011; 2 (2): 205-212.

6. Kawamura H, Bourne R. Factors affecting range of flexion after total knee arthroplasty. J Orthop Sci. 2001; 6 (3): 248-252.

7. Rand A. Modular augments in revision total knee arthroplasty. Orthop Clin North Am. 1998; 29: 347-350.

8. Morrey B. Factors affecting flexion after total knee arthroplasty. Yearbook of Orthopedics. 2008, p. 116.

9. Vera-Avilés FA, Negrete-Corona J, Jiménez-Aquino JM. Artroplastia total de rodilla, pronóstico al restablecer la línea articular. Acta Ortopédica Mexicana. 2012; 26 (6): 362-368.

10. Gutiérrez-García JA, Sierra-Pérez M, García-Velazco RA. Artroplastia total cementada de rodilla: comparación entre el uso o no de isquemia en el resultado postoperatorio inmediato. Acta Ortopédica Mexicana. 2016; 30 (1): 7-12.

11. Blanco F, Fernández-Sueiro J. Artrosis: concepto, clasificación epidemiología y patogenia. En: Reumatología: enfermedades del aparato locomotor, Vol. 1. Madrid: Aran; 1998.

12. Bourne R, Chesworth B, Davis A. Comparing patient outcomes after THA and TKA: is there a difference? Clin Orthop Relat Res. 2009; 468 (2): 542-546.

13. Association of American Retired Persons. Joint Replacement Surgery Patient Survey. Boston. MA, 2007.
14. Rothman R, Parvizi J, Mortazavi S. Secondary resurfacing of the patella after primary TKA: does the anterior knee pain resolve? J Arthroplasty. 2010; 25 (3): e37.

15. Centers for Disease Control and Prevention (CDC). Prevalence of disabilities and associated health conditions among adults: United States. MMWR Morb Mortal Wkly Rep. 2001; 50: 120-125.

16. Srikanth V, Fryer J, Zhai G. A meta-analysis of sex differences prevalence, incidence and severity of osteoarthritis. Osteoarthritis Cartilage. 2005; 13 (9): 769-781.

17. Sharma L. Osteoarthritis year in review 2015: clinical. Osteoarthritis Cartilage. 2016; 24 (1): 36-48.

18. McWilliams D, Leeb B, Muthuri S. Occupational risk factors for osteoarthritis of the knee: a meta-analysis. Osteoarthritis Cartilage. 2011; 19 (7): 829-839.

19. Murphy L, Helmick C. The impact of osteoarthritis in the United States. Orthopaedic Nursing. 2012; 31 (2): 85-91.

20. Alford J, Cole B. Cartilage restoration, part 2: techniques, outcomes, and future directions. Am J Sports Med. 2005; 33 (3): $443-460$.

21. Dillon C, Hirsch R, Rasch E. Symptomatic hand osteoarthritis in the United States. American Journal of Physical Medicine \& Rehabilitation. 2007; 86 (1): 12-21.

22. Treatment of Osteoarthritis of the Knee Guideline [Internet]. American Academy of Orthopaedic Surgeons. 2012 [cited 6 July 2016]. Available from: http://www.aaos.org/research/ guidelines/TreatmentofOsteoarthritisoftheKneeGuideline. pdf.

23. Mahmoudian A, Van Assche D, Herzog W. Towards secondary prevention of early knee osteoarthritis. RMD Open. 2018; 4 (2): e000468.

24. Guía de Práctica Clínica: diagnóstico y tratamiento de la osteoartrosis de rodilla [Internet]. CENETEC. 2009 [Cited 6 June 2016]. Available from: http://www.cenetec.salud.gob.mx/ interior/gpc.html.

25. Cooper C, Adachi J, Bardin T. How to define responders in osteoarthritis. Curr Med Res Opin. 2013; 29 (6): 719-729.

26. Parvizi JM, Lajam CM, Trousdale RT. Total knee arthroplasty in young patients with juvenile rheumatoid arthritis. J Bone Joint Surg Am. 2003; 85-A: 1090-1094.

27. Sultan PG, Most E, Schule S. Optimizing flexion after total knee arthroplasty: advances in prosthetic design. Clin Orthop. 2003; (416): 167-173.

28. Varady NH, Grodzinsky AJ. Osteoarthritis year in review 2015: Mechanics. Osteoarthritis Cartilage 2016; 24 (1): 27-35.

29. Sharkey P, Hozack W, Rothman R. Why are total knee arthroplasties failing today? Clinical Orthopaedics and Related Research. 2002; 404: 7-13.

30. Noble PC, Gordon MJ, Weiss JM. Does total knee replacement restore normal knee function? Clin Orthop Relat Res. 2005; 431: $157-165$

31. Victor J, Bellemans J. Physiologic kinematics as a concept for better flexion in TKA. Clinical Orthopaedics and Related Research. 2006; (452): 53-58.

32. Attune Knee System-Value Analysis Brief [Internet]. http:// www.synthes.com/. 2016 [cited 11 June 2016]. Available from: http://synthes.vo.llnwd.net/o16/LLNWMB8/US\%20Mobile/ Synthes\%20North\%20America/Product\%20Support $\% 20$ Materials/Product\%20Information \%20Sheets/DSUS JRC-0514-0188\%20Val\%20Brief.pdf.

33. Keenan A, Wood A, Arthur C. Ten-year survival of cemented total knee replacement in patients aged less than 55 years. J Bone Joint Surg Br. 2012; 94-B (7): 928-931.

34. Breugem S. Anterior knee pain after a total knee arthroplasty: what can cause this pain? World J Orthopedics. 2014; 5 (3): 163-170. 
35. Sánchez MM, Vasallo JY, Vega A. Manual socot, cirugía ortopédica artrosis de rodilla. 3ra ed. México: McGraw-Hill Interamericana 2002-2004. p. 649-657.

36. Barlow B, Oi K, Lee Y. Incidence, indications, outcomes, and survivorship of stems in primary total knee arthroplasty. Knee Surg Sports Traumatol Arthrosc. 2017; 25 (11): 36113619 .

37. Slover J, Md, Espehaug BM, Phd. Havelin, Md. Costeffectiveness of unicompartmental and total knee arthroplasty in elderly low-demand patients. A markov decision analysis. J Bone Joint Surg. 2006; 88 (11): 2348-2355.

38. Clatworthy M. Outcome \& survival analysis of conventional measured resection, neutral alignment attune TKA vs CAS anatomic tibia, balanced femur, constitutional alignment attune TKA. Orthop J Sports Med. 2017; 5 (5 suppl 5): $2325967117 \mathrm{~S} 0015$

39. What is the KOOS? [Internet]. Koos.nu. 2016 [Cited 27 May 2016]. Available from: http://www.koos.nu/koospresentation. html.
40. Ware J, Owens B, Akelman M. Preoperative KOOS and SF36 scores are associated with the development of symptomatic knee osteoarthritis at 7 years after anterior cruciate ligament reconstruction. Am J Sports Med. 2018; 46 (4): 869-875.

41. Roos E, Lohmander L. The knee injury and osteoarthritis outcome score (KOOS): from joint injury to osteoarthritis. Health Quality Life Outcomes. 2003; 1 (1): 64.

42. Lyman S, Lee Y, Franklin P. Validation of the KOOS, JR. A short-form knee arthroplasty outcomes survey. Clin Orthop Rel Res. 2016; 474 (6): 1461-1471.

43. Carey B, Harty J. A comparison of clinical- and patientreported outcomes of the cemented ATTUNE and PFC sigma fixed bearing cruciate sacrificing knee systems in patients who underwent total knee replacement with both prostheses in opposite knees. J Orthop Surg Res. 2018; 13 (1): 54.

44. Ranawat C, White P, West S, Ranawat A. Clinical and radiographic results of attune and $\mathrm{PFC}$ sigma knee designs at 2-year follow-up: a prospective matched-pair analysis. J Arthroplasty. 2017; 32 (2): 431-436. 